Gbekley et al., Afr J Tradit Complement Altern Med., (2018) 15 (1): $85-97$

https://doi.org/10.21010/ajtcam.v15i1.9

\title{
ETHNOBOTANICAL SURVEY OF MEDICINAL PLANTS USED IN THE MANAGEMENT OF HYPERTENSION IN THE MARITIME REGION OF TOGO
}

\author{
Holaly E. Gbekley ${ }^{1}$, Simplice D. Karou ${ }^{1,3^{*}}$, Gnatoulma Katawa ${ }^{1}$, Tchadjobo Tchacondo ${ }^{1}$, Komlan \\ Batawila $^{2}$, Yaovi Ameyapoh ${ }^{1}$, Jacques Simpore ${ }^{3}$
}

${ }^{1}$ Ecole Supérieure des Techniques Biologiques et Alimentaires (ESTBA-UL), Université de Lomé, Togo; ${ }^{2}$ Laboratoire de Botanique et Biologie Végétale, Faculté des Sciences (FDS-UL), Université de Lomé, Togo. Laboratoire de Biologie et Ecologie Végétales, Faculté des Sciences, Université de Lomé; ${ }^{3}$ Centre de Recherche Biomoléculaire Pietro Annigoni (CERBA/LABIOGENE), Ouagadougou, Burkina Faso

*Corresponding Author Email: simplicekarou@ @otmail.com

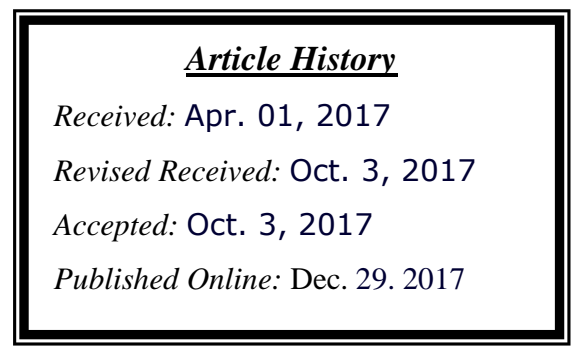

\begin{abstract}
Background: Hypertension is one of the most common chronic diseases in modern societies and there is evidence that its incidence and severity are increasing. This survey was conducted to investigate the plants used by the Togolese traditional healers to treat the disease.

Method: From January to June 2016, an ethnobotanical survey was conducted using a semi-structured questionnaire with traditional healers $(\mathrm{TH})$ in the southern region of Togo. The importance of the plants was assessed by the calculation of the use value (UV).

Results: In Total, $128 \mathrm{TH}$ male and female were interviewed and 116 plants species belonging to 46 families were identified as treating hypertension. The most represented families were: Fabaceae with 16 species followed by Euphorbiaceae and Rutaceae contributing with 8 and 6 species respectively. Based on the calculated use values the most important species were Byrsocarpus coccineus Schum. Et thonn. (UV = 0.47); Crateva religiosa G.Forst. (UV = 0.47), Boerhavia diffusa Engelm. \&A.Gray L. (UV = 0.47), Xylopia aethiopica A. Rich. (UV = 0.42), Mangnifera indica $\mathrm{L}$. $(\mathrm{UV}=0.38)$. The leaves and the roots were the parts of plant predominantly used to prepare the recipes, mainly decoctions administrated by oral route. Clinical manifestations such as dizzy spells, swarming, loss of consciousness, severe headache, severe anxiety and shortness of breath, nosebleed, and fear of heights were used by $\mathrm{TH}$ to diagnose the disease.

Conclusion: This study showed initial evidence of the use of plant materials by Togolese TH to treat hypertension. These results constitute a database for pharmacological screenings with the aim of developing new therapies.
\end{abstract}

Key words: Hypertension, traditional medicine, ethnobotanical survey, medicinal plants, Togo.

\section{Introduction}

Nowadays, there is a renewed interest in medicinal plants research because of health problems that remained unsolved (Briskin, 2000; Rafieian-Kopaei, 2011; Atanasov et al., 2015). The main examples are infectious disease such as malaria (Larremore et al., 2015; Rosa et al., 2015) and bacterial infections (Cohen et al., 2015; Le Doare et al., 2015; Oneko et al., 2015).To these diseases are added cancer and metabolic diseases such as diabetes and arterial hypertension whose incidence is increasing (Frohlich, 2001; Sowers et al., 2009). Hypertension is generally a chronic condition and is often associated with few or no symptoms. Symptoms usually occur when blood pressure spikes suddenly and extremely enough to be considered as a medical emergency. Rare symptoms include dizzy spells, headaches, and nosebleeds (Svilaas et al., 2008). Many people may suffer from the disease without knowing it. Uncontrolled high blood pressure, or hypertension, causes damage to arteries. It is also a risk factor for stroke, heart attack, and other cardiovascular problems.

There are a number of treatments for high blood pressure, ranging from lifestyle changes, weight loss, and medication. Many efforts are being made in synthetic chemistry to bring to market new drugs against the disease, but the need for new molecules arises today with acuity. This current situation justifies the new resurgence of interest in medicinal plants, given their potential in this matter (Gali-Muhtasib et al., 2015; Baharvand-Ahmadi and Asadi- 
Samani, 2016). In the particular case of hypertension, there is a high prevalence of usage of complementary medicine. Indeed, plants have always contributed largely in the fight against various diseases, considering the number of remedies derived directly or indirectly from plants (Dzib-Guerra et al., 2016; Rouhi-Boroujeni et al., 2016). Consequently, in the recent decades, the medicinal plants used in the management of the disease have attracted the attention of some authors, through the screening for loss level blood pressure activity of plant extracts.

In Africa, it is a question of culture and tradition and it is estimated that over $80 \%$ of the population in rural areas have exclusive use of plants for their primary health care needs (Baharvand-Ahmadi et al., 2016). Increasingly, studies related to medicinal plants are conducted by researchers in Africa and some lead to the identification of active principles (Tchacondo et al., 2012; Ilboudo et al., 2013). However, data on the ethnobotany of plants used in the management of Hypertension are scanty. In the particular case of Togo, Karou et al. (2011) conducted a study of the plants used in the treatment of diabetes and hypertension in the central region, one of five regions in the country. Data are missing for the rest of the country. The present study was undertaken to investigate the treatment of Hypertension by traditional healers in the Southern region of Togo.

\section{Materials and Methods Study area}

Togo is a western African country lying between Burkina Faso in the North, Benin in the East, Ghana in the West and the Atlantic Ocean in the South. The country is divided into five economic regions namely Savannah Region, Kara Region, Central Region, Plateau Region, and Maritime Region. The present study was carried out in the Maritime Region (figure 1). It stands between $1^{\circ} 20^{\prime}-1^{\circ} 50^{\prime}$ east and $6^{\circ} 10^{\prime}-6^{\circ} 60^{\prime}$ north of the equator and bordered to the north, West, East and the South by Plateau Region, Republic of Ghana, Republic of Benin and the Atlantic Ocean respectively. This study area is $6100 \mathrm{~km}^{2}$ big and occupies approximately $10.78 \%$ of the country. The climate is subequatorial. The region is inhabited by 1.828 .000 people (density of $50-200$ persons $/ \mathrm{km}^{2}$ ), the main ethnic groups being Ewe, Ouatchi, Mina, Fon, Adja.

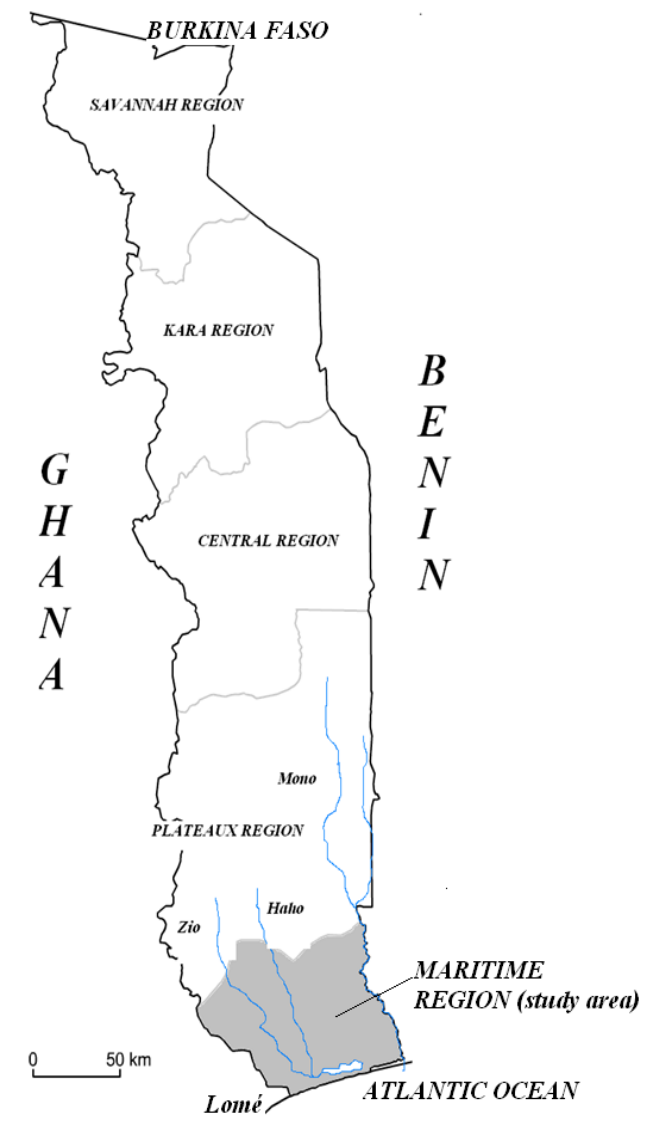

Figure 1: Map of Togo showing the Maritime Region

\section{Data collection}

Direct interviews with traditional healers (TH) were conducted between June and August 2016 using a semistructured questionnaire. Each TH gave a verbal consent certifying his/her agreement with the form issued to explain the importance of the information they would provide prior to interviews. Questions asked were about (i) the $\mathrm{TH}$ identity, i.e. name and surname, sex, age, level of education; (ii) the origin of their knowledge; (iii) the status of the TH, 
i.e. full-time professional $\mathrm{TH}$ or part-time professional $\mathrm{TH}$; (iv) the disease, i.e. name of the disease in the local language; (v) the diagnosis, i.e. main symptoms; and (vi) the remedies, i.e. the number of plants in the remedy, the local names of the plants, the used parts, the mode of preparation, and the administration route.

\section{Plant identification}

After interviews, preliminary identification of the plants was done in the field by a botanist. Afterward, herbarium specimens were prepared and pictures were taken to help in the confirmation of the identity of the plants. Plant identities were confirmed by comparison with available voucher specimens in the Herbarium of the Botany Department, University of Lomé, using taxonomic keys of online databases of West African Plants - A photo Guide on the website: http://www.westafricanplants.senckenberg.de/root/index.php. Nomenclature of species was done using the online data base of IPNI website: http://www.ipni.org/ipni/plantnamesearchpage.do.

\section{Statistical analysis}

Excel spread sheet was used to make simple calculations and to determine plant frequencies. The use value (UV), a quantitative method that demonstrates the relative importance of species known locally, was calculated according to the following formula (Aburjai et al., 2007; Hudaib et al., 2008):

$\mathrm{UV}=\Sigma \mathrm{U} / \mathrm{n}$

where, UV is the use value of a species; $\Sigma \mathrm{U}$ the total number of citations per species; $\mathrm{n}$ the number of informants. The other analyses were performed using PRISM 5.02 program (GraphPad Software, Inc., La Jolla, USA). Since most of the variables did not show a normal distribution, the following tests were chosen: to compare three groups a KruskalWallis-test was performed and, if significant, followed by a Mann-Whitney-U test for a further comparison of the groups. P-values of 0.05 or less were considered significant.

\section{Results}

\section{Socio-demographic profile of the traditional healers}

One hundred and twenty-eight traditional healers ( 83 males and 45 females) were interviewed in the present study. Table 1 displays the socio demographic profile of the TH. The TH were divided into five age groups, notably the less than 30 years, 30 to 50, 50 to 70,70 to 90 and the more than 90 years. According to the recorded data on the $\mathrm{TH}$ implicated in the treatment of arterial hypertension in the surveyed region, the 30 years and 70 to 90 years old individuals accounting respectively for 9.38 and $31.25 \%$ were less represented compared to either 30-50 years or 50-70 years old people. Only 2 TH were above 90 years. With regards to the ethnic groups the majority belonged to the native ethnics of the region namely the Ewe $(50.00 \%)$ and Mina $(25.00 \%)$. Three religions were recorded; the Animists, the Christian and the Muslims, but the most represented were the Animists (43.75\%) and the Christians (41.41\%). Concerning the educational level, the TH could be ranged the following groups: the illiterates, the primary school level, the secondary school level and the university level, with respective contributions of 23.44, 39.06, 31.25 and $6.25 \%$. The TH who attended primary school level were more compared to secondary school $(\mathrm{p}=0.0003)$ and university level. The familial heritance, the initiation from a senior $\mathrm{TH}$ and the divine revelation were the recorded means of transmission of the medicinal practice among the surveyed TH. Our results indicated that the family inheritance accounting for $42.97 \%$ of $\mathrm{TH}$ was the most common mean for the transmission of the knowledge, followed by the divine revelation and the traditional $(\mathrm{p}<0.0001)$. The $\mathrm{TH}$ in the formal sector $(44.53 \%)$, thus exerting the traditional medicine as a secondary occupation were more represented in the Hypertension treatment compared to either artisans $(\mathrm{p}<0.0001)$ or farmers $(\mathrm{p}=0.0002)$. Only $22.66 \% \mathrm{TH}$ exerted the traditional medicine as the unique source of revenue. Thus we could find that for the payment, the majority $(49.22 \%)$ of them receives the payment after pain relief but an important portion $(35.16 \%)$ receives the payment before the treatment. Some of the TH $(23.44 \%)$ argued they collaborate with the modern medicine by referring the most complicated cases, the other do not collaborate.

Table 1 : Socio-demographic profile of the surveyed traditional healers

\begin{tabular}{lll}
\hline Characteristics & Groups & $\begin{array}{l}\text { Respondents } \\
\mathrm{N}(\%)\end{array}$ \\
\hline Gender & Males & $83(64.84)$ \\
& Females & $45(35.16)$ \\
Age (years) & $<30$ & $12(9.38)$ \\
& {$[30-50[$} & $40(31.25)$ \\
& {$[50-70[$} & $54(42.19)$ \\
Ethnicity & {$[70-90[$} & $20(15.63)$ \\
& $>90$ & $2(1.56)$ \\
& Ewe & $64(50.00)$ \\
\hline
\end{tabular}




\begin{tabular}{lll}
\hline \multirow{2}{*}{ Religion } & Other & $32(25.00)$ \\
& Animists & $56(43.75)$ \\
& Christians & $53(41.41)$ \\
& Muslims & $19(14.84)$ \\
& Illiterates & $30(23.44)$ \\
& Elementary school & $50(39.06)$ \\
Origin of the knowledge & Secondary school & $40(31.25)$ \\
& University & $8(6.25)$ \\
Collaboration with modern medicine & Familial heritance & $55(42.97)$ \\
& Initiation from a TH & $36(28.13)$ \\
Professional status of the TH & Divine revelation & $37(28.91)$ \\
& Collaborative & $30(23.44)$ \\
& Non collaborative & $98(76.56)$ \\
& Full time profession & $29(22.66)$ \\
& Farmer & $31(24.22)$ \\
Honoraria & Artisans & $11(8.59)$ \\
& Formal sector & $57(44.53)$ \\
& Before the treatment & $45(35.16)$ \\
& During the treatment & $20(15.63)$ \\
\hline
\end{tabular}

\section{Symptoms used by TH for the diagnosis of High Blood Pressure}

A total of 8 symptoms were identified (table 2). A particular symptom was not used alone to identified the disease, hence a TH could cited more than two symptoms in the diagnosis of the disease. According to table 2 , all the TH cited the nosebleeds as the main symptom of hypertension. In the order of importance the other symptoms were ranged as follows: Headache, swarming, loss of consciousness, dizzy spells, anxiety, shortness of breath and the fear of height.

Table 2: The symptoms of hypertension cited by the surveyed traditional healers

\begin{tabular}{ll}
\hline Symptoms & $\begin{array}{l}\text { Respondents } \\
\text { N }(\%)\end{array}$ \\
\hline Nosebleeds & $128(100.00)$ \\
Headache & $118(92.19)$ \\
Swarming & $112(87.50)$ \\
Loss of consciousness & $111(86.72)$ \\
Dizzy spells & $107(83.60)$ \\
Anxiety & $92(71.86)$ \\
Shortness of breath & $75(58.59)$ \\
Fear of height & $67(52.34)$ \\
\hline
\end{tabular}

\section{Diversity of medicinal plants and their usage in the treatment of Hypertensionin the maritime region}

A total of 116 plants species ranged in 46 families were recorded in the present study. The plants consisted of trees, herbs, lianas and shrubs; the most frequent growth habits being the trees and shrubs. They accounted for $56.41 \%$ and $22.05 \%$ respectively (figure 2 ). The species were diversely distributed among botanical families. Thus, some families were more represented than others. The most represented family was the Fabaceae that contributed with 16 species namely Baphia nitida Lodd., Indigofera pulchra Willd., Milletia thonningii Baker., Parkia biglobosa Benth., Pericopsis laxiflora Benth. Ex Baker Meeuwen, Xeroderris stuhlmannii Taub Mendoça \& E.P. Sousa , Callindra haematocephala, Afzelia Africana Sm., Cassia occidentalis L. , Senna hirsute, Piliostigma thonningii (Schum.) Milne-Redh. , Senna occidentalis (L.) Link ,Senna alata(L.) Roxb,), Albizia adianthifolia schumwhight, Uraria picta , Afrormosia laxiflora Benth. Exbak. Harms. This was followed by Euphorbiaceae contributing with 8 species (Croton lobatus L., Elophorbia grandifolia (Haw.) Croizat, Euphorbia hirta L., Jatropha curcas L., Jatropha gossypiifolia L., Phyllanthus amarus Schum., Ricinus communis L. and Securinega virosa Willd. Baill.) and Rutaceae with 6 species(Citrus aurantifolia (Christm.) Swingle, Citrus aurantium L., Citrus grandis Hassk., Clausena anisata (Willd.) Hook.f. ex Benth., Fagara macrophylla Engl., and Zanthoxylum zanthoxyloides (Lam.) Zepern. \& Timler). The other families contributed with less than 5 species. 


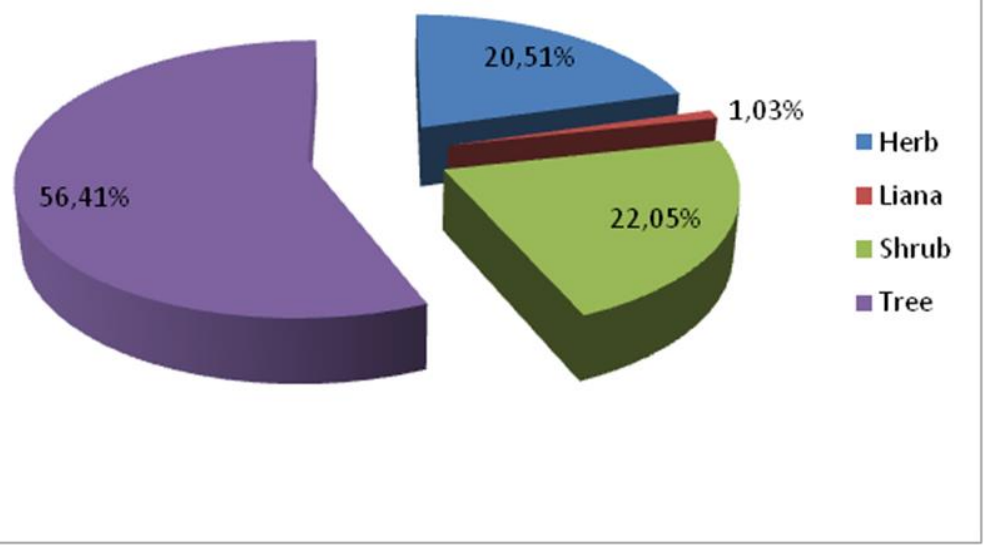

Figure 1 : Growth habits of medicinal plants used in the management of hypertension

The importance of medicinal plants was assayed by the calculated use values (UV) that were ranged between 0.05 for the less used species and 0.47 for the most used species (table 3 ). Considering these UV, the following species appeared to be of great importance for the management of hypertension in the surveyed region: Byrsocarpus coccineus Schum. Etthonn. (UV = 0.47); Crateva religiosa G. Forst. (UV = 0.47), Boerhavia diffusa Engelm. \&A.Gray L. (UV =0.47), Xylopia aethiopica A. Rich. (UV =0.42), Mangnifera indica L. (UV =0.38).

The TH in the maritime region of Togo were found to use various parts of plant in the treatment of hypertension. Figure 3 displays the trends of use of the various plants parts. The leaves $(52.63 \%)$ are the main parts of plants used in the preparations of recipes for the treatment of High Blood Pressure.

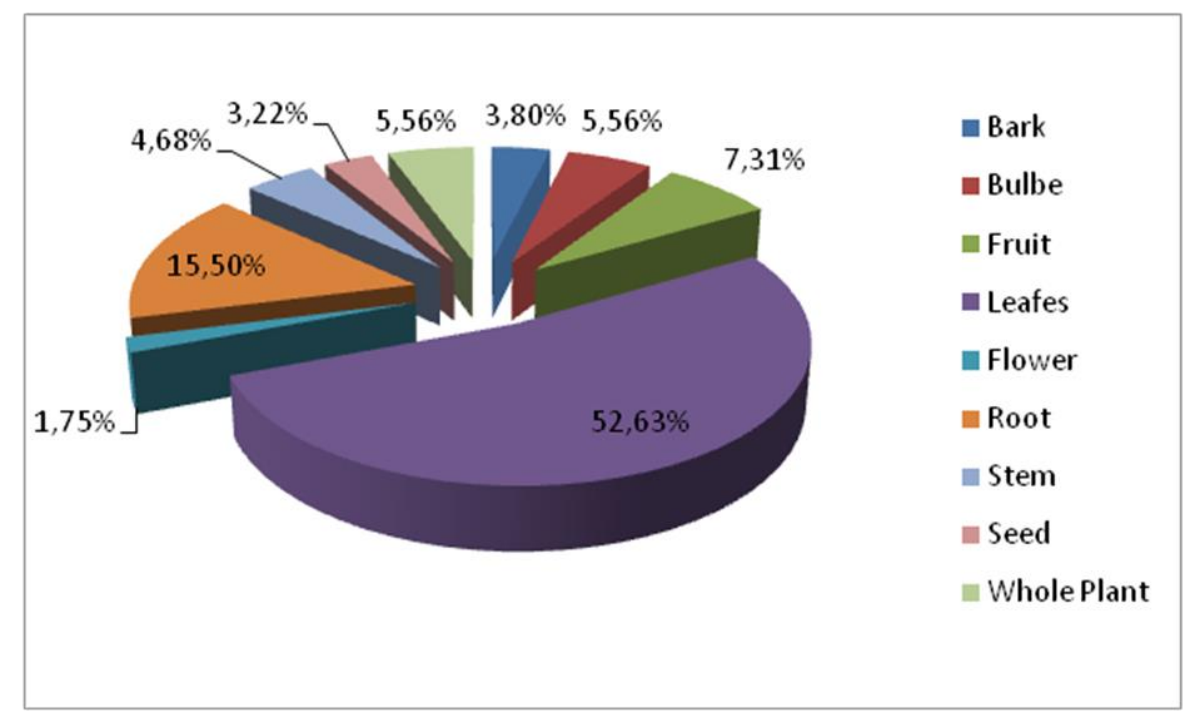

Figure 2 : Parts of the plants used for the treatment of High Blood Pressure.

A total of 7 modes of preparation including decoction, infusion, powder, tea, crushing, alcoholic maceration and juice, were identified. Decoctions were obtained by boiling either fresh or dried materials in water. However the amount and the duration varied from one TH to another. In some cases, the TH could give the prepared decoction or in other cases the TH gives the plant materials and the indications to prepare the recipes. Crushing was obtained from fresh materials by direct pounding in a mortar and the filtration through a tissue and the filtrate could be directly administrated in the appropriate dose by oral route. The juice from Rutaceae was obtained by pressure of the fruit. The tea was obtained by maceration of dried materials in water for the appropriate time and administrated by oral route. Only one case of alcohol maceration was recorded in the case of Chenopodium ambrosiodes L. This is prepared by maceration of the powder of leaves in the traditionally distillated liquor from palm wine locally known as Sodabi. In some cases, there are additives such as honey, or milk the TH recommend the use. According to our results, decoctions were more used by TH compared to the powder $(\mathrm{p}=0.15)$. Both were predominantly used by TH compared to other formulations (Figure 4). 


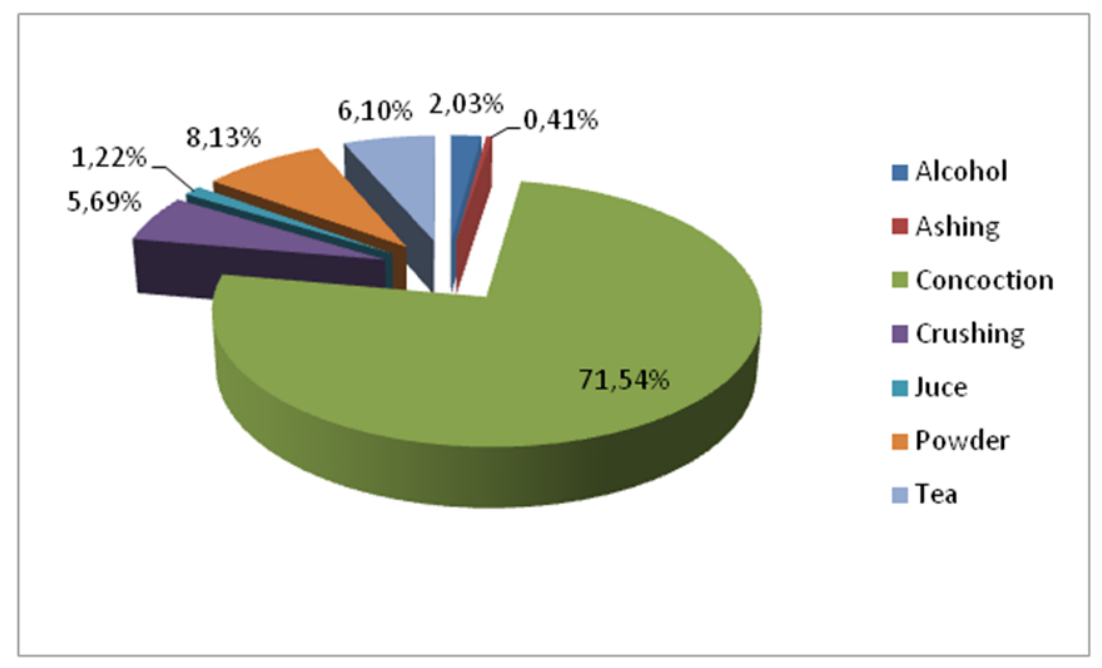

Figure 3 : Mode of preparation of recipes used in the management of hypertension

As indicated in figure 5, most of the formulations were administrated by the oral route $(91.06 \%, \mathrm{p}<0.0001)$. This was followed by bath (5.28\%).In contrast, few TH preferred bandage, massage, poultice, cataplasm and smoking.

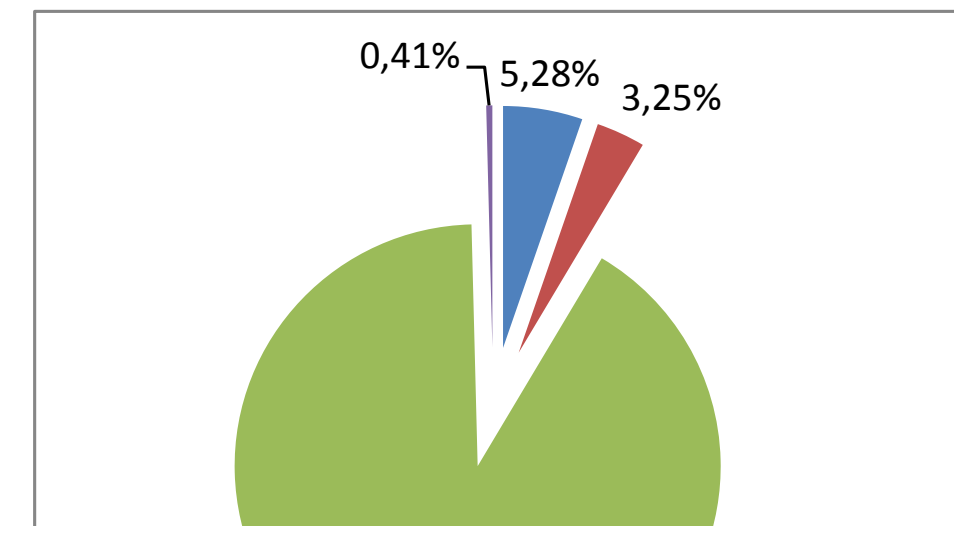

Figure 4 : Administration route of medicinal recipes used in the management of hypertension 
Table 3: Diversity of medicinal plants and their uses for the treatment of liver damage in the Maritime Region

\begin{tabular}{|c|c|c|c|c|c|c|c|c|c|}
\hline Family & Species & Local name & Voucher $\mathrm{N}^{\circ}$ & UV & $\begin{array}{l}\text { Used } \\
\text { parts }\end{array}$ & Habits & $\begin{array}{l}\text { Mode of } \\
\text { preparation }\end{array}$ & Route & Previous citation \\
\hline \multirow[t]{2}{*}{ Amaranthaceae } & Gomphrena celosioides C. Mart. & Amegatahe & Togo 01731 & 0,09 & WP & Herb & Dec, Inf & oral & (Adjanohoun et al., 1991) \\
\hline & Aerva lanata (L.) A. L. Juss. & Eweowo & - & 0,05 & WP & Tree & Inf & Oral, & $\begin{array}{l}\text { (Adjanohoun et al., 1991) } \\
\text { (Cisse et al., 2016) }\end{array}$ \\
\hline \multirow[t]{3}{*}{ Anacardiaceae } & Sclerocarya birrea (A.Rich.) Hochst. & - & 1828 & 0,09 & $\mathrm{Fl}$ & Tree & Dec. & Oral & (Adjanohoun et al., 1991) \\
\hline & Anarcadium occidentale $\mathrm{L}$. & Yovotsan & Togo 01768 & 0,19 & St & Tree & Dec. & Oral, & $\begin{array}{l}\text { (Tchacondo et al., 2011; } \\
\text { Karou et al., 2011; Tra Bi et } \\
\text { al., 2008 ; Tokoudagba et al., } \\
\text { 2009; Kayode, 2006) }\end{array}$ \\
\hline & Mangnifera indica $\mathrm{L}$. & Amangoti & 1157FDS/UL & 0,38 & Lf & Tree & Dec & oral & (Cisse et al., 2016) \\
\hline \multirow[t]{3}{*}{ Annonaceae } & Annona senegalensis Pers. & Dzogbenyikli & Togo 01881 & 0,09 & Le & Tree & Dec. & Oral, & (Karou et al., 2011) \\
\hline & Xylopia aethiopica A. Rich. & Etso & 1987 & 0,42 & St & Tree & Dec. & Oral & $\begin{array}{l}\text { (Karou et al., 2011; } \\
\text { Tokoudagba et al., 2009) }\end{array}$ \\
\hline & Zea maïs L. & Ebli & - & 0,09 & Fr & Tree & Dec. & Oral & $\begin{array}{l}\text { (Orch et al., } 2015 \text {; Sereme et } \\
\text { al., 2008) }\end{array}$ \\
\hline \multirow[t]{4}{*}{ Apocynaceae } & Catharanthus roseus L. G.Don & Flawavigbé & 95FDS/UL & 0,09 & Lf & Shrub & Dec. & Oral, & $\begin{array}{l}\text { (Karou et al., } 2011 \text {; Tra Bi et } \\
\text { al., 2008) }\end{array}$ \\
\hline & Picralima nitida Th. \&H.Dur. & Ayokpè & 2105/220FDS/UL & 0,09 & $\mathrm{Sd}$ & Shrub & Dec. & Oral & (Karou et al., 2011) \\
\hline & Rauvolfia vomitoria Afzl. & Dodemakpowoe & TG12750 & 0,05 & $\mathrm{Bk}$ & Shrub & Dec, Pow & Oral & $\begin{array}{l}\text { (Adjanohoun et al., } 1991 \text {; } \\
\text { Padal et al., 2010) }\end{array}$ \\
\hline & Strophantus hispidus & $\begin{array}{l}\text { Sagere/dikuyinti } \\
\mathrm{n}\end{array}$ & - & 0,05 & Rt & Herb & Dec. & Oral & (Adjanohoun et al., 1991) \\
\hline \multirow[t]{2}{*}{ Arecaceae } & Cocos nucifera $\mathrm{L}$. & Netsi & 02481TG Clt/AK & 0,09 & Rt & Tre & Dec & Oral & (Karou et al., 2011) \\
\hline & $\begin{array}{l}\text { Raphia hookeri G. Mann \& H. } \\
\text { Wendl. }\end{array}$ & Plamploti-ma & 470FDS/UL & 0,09 & $\mathrm{Fl}$ & Herb & Dec, Cru & Oral & (Adjanohoun et al., 1991) \\
\hline Asclepiadaceae & Pergularia aemia & - & 2314 & 0,09 & Lf & Tree & Dec. & Bath & (Adjanohoun et al., 1991) \\
\hline \multirow[t]{4}{*}{ Asteraceae } & Vernonia amygdalina Delile & $\begin{array}{l}\text { Aluma/Gbondut } \\
\text { si }\end{array}$ & Togo 01204 & 0,09 & Le & Shrub & Dec, Pow & Oral & (Karou et al., 2011) \\
\hline & Vernonia cinerea (L.) Less. & Hunssikonou & - & 0,09 & Lf & Shrub & Dec & Oral & $\begin{array}{l}\text { (N'Guessan et al., } 2011 \text {; } \\
\text { Karou et al., 2011; Tra Bi et } \\
\text { al., 2008) }\end{array}$ \\
\hline & Acanthospermum hispidum DC. & Apegbin & 00749TGClt/AK & 0,28 & Le & Herb & Dec & Oral, & (Tokoudagba et al., 2009) \\
\hline & Bidens pilosa Linn. & - & 841 & 0,09 & Lf & Tree & Dec. & Oral, & (Dibong et al., 1997) \\
\hline \multirow[t]{3}{*}{ Bignoniaceae } & Kigelia Africana (Lam.) Benth. & Gnakpekpe & $1816^{*}$ & 0,05 & $\mathrm{Bk}$ & Tree & Dec. & oral & (Adjanohoun et al., 1991) \\
\hline & $\begin{array}{l}\text { Newbouldia laevis (P. Beauv.) } \\
\text { Seeman ex. Bureau }\end{array}$ & Kpatsima & 337FDS/UL & 0,19 & Le & Tree & Dec. & oral & (Adodo, 2004) \\
\hline & Stereospermum kunthianum Cham. & & - & 0,05 & St & Herb & Dec. & Oral & (Adjanohoun et al., 1991) \\
\hline \multirow[t]{2}{*}{ Bombacaceae } & Adansonia digitata $\mathrm{L}$ & Adidotima & 1190FDS/UL & 0,19 & Le & Tree & Pow & Oral, & (Tra Bi et al., 2008) \\
\hline & Ceiba pentandra L. Gaerth. & - & 2485 & 0,09 & Lf & Tree & Dec. & Oral, & (Tra Bi et al., 2008) \\
\hline Boraginaceae & Heliotropium indicum $\mathrm{L}$. & $\begin{array}{l}\text { KoklotadoèAga } \\
\text { mashike }\end{array}$ & Togo 02508 & 0,05 & Lf & Dec/Orl & Dec. & oral & (Tokoudagba et al., 2009) \\
\hline Capparaceae & Crateva religiosa G. Forst. & Awatayisan & 00326TGClt/AK & 0,47 & $\mathrm{Bk}$ & Tree & Dec & Oral & (Tokoudagba et al., 2009) \\
\hline Chenopodiaceae & Chenopodium ambrosiodes L. & Arunpale & - & 0,05 & Lf & Shrub & alc & Oral, & (Orch et al., 2015) \\
\hline
\end{tabular}




\begin{tabular}{|c|c|c|c|c|c|c|c|c|c|}
\hline Connaraceae & $\begin{array}{l}\text { Byrsocarpus coccineus Schum. Et } \\
\text { thonn. }\end{array}$ & tomégavigbé & 12603 & 0,47 & $\mathrm{Lf}$ & Tree & Tea & Oral, & (Tokoudagba et al., 2009) \\
\hline \multirow[t]{2}{*}{ Cucurbitaceae } & Momordica charantia L. & Agnagnra & 2799/6182 FDS/UL & 0,09 & WP & Liana & Dec, Pow & oral & $\begin{array}{l}\text { (Karou et al., } 2011 \text {; Tra Bi et } \\
\text { al., 2008) }\end{array}$ \\
\hline & Adenopus breviflorus & Tagiri & - & 0,05 & Fr & Tree & Cru & Oral, & (Adjanohoun et al., 1991) \\
\hline Dracaenaceae & Dracaena arborea & Anyantsi & Togo 09453 & 0,05 & Le & Tree & Dec & Oral & (Ajibesin et al., 2008) \\
\hline Ebenaceae & Diospyrosmes piliformis Hochst. & Tigbado & 672FDS/UL & 0,38 & $\mathrm{TF}$ & Tree & Dec & Oral & (Karou et al., 2011) \\
\hline \multirow{8}{*}{ Euphorbiaceae } & Croton lobatus $L$. & Eru & - & 0,05 & Fr & Tree & Dec & Oral & (Adjanohoun et al., 1991) \\
\hline & $\begin{array}{l}\text { Elophorbia grandifolia (Haw.) } \\
\text { Croizat }\end{array}$ & - & - & 0,19 & WP & Tree & Dec & Oral & (Adjanohoun et al., 1991) \\
\hline & Euphorbia hirta L. & Hundihundi & 454FDS/UL & 0,19 & Le & Shrub & Dec & Smoking & (Tra Bi et al., 2008) \\
\hline & Jatropha curcas $\mathrm{L}$. & Babatihé & - & 0,19 & Lf & Tree & Dec. & oral & (Tra Bi et al., 2008) \\
\hline & Jatropha gossypiifolia L. & Babatidzin & - & 0,19 & Lf & Tree & Dec. & oral & (Tokoudagba et al., 2009) \\
\hline & Phyllanthus amarus Schum. & Ahlivi & Togo 03349 & 0,09 & WP & Herb & Dec. & Oral & $\begin{array}{l}\text { (Tchacondo et al., } 2011 \text {; Tra } \\
\text { Bi et al., 2008) }\end{array}$ \\
\hline & Ricinus communis $\mathrm{L}$. & Kassouwèlti & 3729 & 0,09 & $\mathrm{Fl}$ & Shrub & Dec. & Oral & $\begin{array}{l}\text { (Karou et al., } 2011 \text {; Tra Bi et } \\
\text { al., 2008) }\end{array}$ \\
\hline & Securinega virosa Willd. Baill. & Hésreé & 3750 & 0,09 & $\mathrm{Fl}$ & Tree & Dec. & Oral & (Adjanohoun et al., 1991) \\
\hline \multirow[t]{10}{*}{ Fabaceae } & Afzelia africana $\mathrm{Sm}$. & Welou & - & 0,09 & $\mathrm{Se}$ & Herb & Dec,Tea & Oral, & (Karou et al., 2011) \\
\hline & Baphia nitida Lodd. & Eto & - & 0,09 & $\mathrm{Bk}$ & Tree & Dec. & Oral, & (Adjanohoun et al., 1991) \\
\hline & Callindra haematocephala & Tude & - & 0,05 & Rt & Herb & Pow & oral & (Adjanohoun et al., 1991) \\
\hline & Indigofera pulchra Willd. & Okamon & Togo 06308 & 0,05 & Lf & Tree & Dec. & oral & (Adjanohoun et al., 1991) \\
\hline & Milletia thonningii Baker. & Kodoliya & 6397 & 0,09 & Rt & Tree & Dec. & oral & (Karou et al., 2011) \\
\hline & Parkia biglobosa Benth. & Ewoati & 329FDS/UL & 0,19 & Lf & Tree & Dec. & oral & $\begin{array}{l}\text { (Karou et al., } 2011 \text {; Tra Bi et } \\
\text { al., 2008 ; Tokoudagba et al., } \\
\text { 2009) }\end{array}$ \\
\hline & Pericopsis laxiflora Benth. & Tchamani & 6492 & 0,09 & Lf & Tree & Dec. & Bath & (Karou et al., 2011) \\
\hline & $\begin{array}{ll}\text { Xeroderris stuhlmannii } & \text { Taub } \\
\text { Mendoça \& E.P.Sousa } & \end{array}$ & Tchalawâri & 6768 & 0,09 & Rt & Tree & Dec. & Oral & (Karou et al., 2011) \\
\hline & Cassia occidentalis $\mathrm{L}$. & Bessisan & 115 & 0,09 & $\mathrm{Se}$ & Shrub & Dec. & Oral, & (Tra Bi et al., 2008) \\
\hline & $\begin{array}{l}\text { Senna hirsute (L.) H.S. Irwin \& } \\
\text { Barneby }\end{array}$ & Madonsohomé & 29 & 0,09 & $\mathrm{Fl}$ & Shrub & Dec. & Oral & (Adjanohoun et al., 1991) \\
\hline Fabaceae / & Piliostigma thonningii (Schum.) & Klo & Togo 0024 & 0,05 & $\mathrm{Lf}$ & Tree & Dec. & Oral & (Karou et al., 2011) \\
\hline \multirow[t]{3}{*}{ Caesalpiniaceae } & Milne-Redh. & & & & & & & & \\
\hline & Senna alata (L.) Roxb,) & Yovologbo & 378FDS/UL & 0,05 & Lf & Shrub & Dec, Pow & Oral & (Adjanohoun et al., 1991) \\
\hline & Senna occidentalis (L.) Link & Bessissan & - & 0,05 & Lf & Herb & Dec. & Oral & (Adjanohoun et al., 1991) \\
\hline & Albizia adianthifolia schumwhight & Agla & 4853137FDS/UL & 0,09 & Le & Herb & Dec & Oral, & (Tra Bi et al., 2008) \\
\hline \multirow[t]{2}{*}{ oideae } & $\begin{array}{l}\text { Afrormosia laxiflora Benth. } \\
\text { Exbak.Harms }\end{array}$ & - & - & 0,09 & $\mathrm{Se}$ & Herb & Dec,Tea & Oral, & (Adjanohoun et al., 1991) \\
\hline & Uraria picta $(\mathrm{Jacq}) \mathrm{DC}$ & - & - & 0,05 & Le & Shrub & Dec. & Oral & (Orch et al., 2015) \\
\hline Hypoxidaceae & $\begin{array}{l}\text { Curculigo pilosa (Schumach. } \\
\text { \&Thonn.) Engl. }\end{array}$ & Epekun & - & 0,05 & WP & Tree & Dec & Oral & (Adjanohoun et al., 1991) \\
\hline Iridaceae & Gladiolus psittacinus Hook. & Baka & - & 0,05 & WP & Herb & Dec & oral & (Adjanohoun et al., 1991) \\
\hline \multirow[t]{2}{*}{ Lamiaceae } & Hyptis suaveolens L. Poit. & Botifadini & - & 0,09 & Lf & Tree & Dec. & oral & (Karou et al., 2011) \\
\hline & Ocimum basilicum L. & Ahameyovoto & 04199TGClt/AK & 0,09 & $\mathrm{Fl}$ & Shrub & Dec & oral & (Karou et al., 2011 ; Orch et \\
\hline
\end{tabular}




\begin{tabular}{|c|c|c|c|c|c|c|c|c|c|}
\hline & Ocimum canum Sims & Ahamè & Togo 04196 & 0,09 & Le & Shrub & Pow & $\begin{array}{l}\text { Massage, } \\
\text { Poultice }\end{array}$ & $\begin{array}{l}\text { al., 2015) } \\
\text { (Karou et al., 2011) }\end{array}$ \\
\hline & Ocimum gratissimum $\mathrm{L}$. & Esrou,Deveti & 1197FDS/UL & 0,19 & WP & Shrub & Dec, Pow & Bath & $\begin{array}{l}\text { (N'Guessan et al., 2011), } \\
\text { (Karou et al., } 2011 \text {; Tra Bi et } \\
\text { al., 2008) }\end{array}$ \\
\hline Lauraceae & Persea americana Mill. & PEYA & - & 0,09 & Lf & Tree & Dec. & Oral & $\begin{array}{l}\text { (Karou et al., } 2011 \text {; Tra Bi et } \\
\text { al., 2008) }\end{array}$ \\
\hline \multirow[t]{2}{*}{ Liliaceae } & Alium cepa $\mathrm{L}$. & sabulè & 300FDS/UL & 0,19 & $\mathrm{Bu}$ & Herb & Tea & Oral, & $\begin{array}{l}\text { (Karou et al., } 2011 \text {; Tra Bi et } \\
\text { al., 2008; Orch et al., } 2015 \text {; } \\
\text { Tokoudagba et al., 2009) }\end{array}$ \\
\hline & Allium sativum $\mathrm{L}$. & Ail, ayo & 296FDS/UL & 0,19 & $\mathrm{Bu}$ & Herb & Pow & Oral, & $\begin{array}{l}\text { (Karou et al., } 2011 \text {; Tra Bi et } \\
\text { al., } 2008 \text {; Orch et al., } 2015 \text {; } \\
\text { Tokoudagba et al., 2009) }\end{array}$ \\
\hline Loganiaceae & Anthocleista djalonensis A. Chev. & Gboloba & 4781 & 0,09 & Rt & Tree & Dec. & Oral, & $\begin{array}{l}\text { (Karou et al., } 2011 \text {; Tra Bi et } \\
\text { al., 2008) }\end{array}$ \\
\hline \multirow[t]{3}{*}{ Malvaceae } & Gossypium arboretum L. & Cotonnier & 693FDS/UL & 0,05 & $\mathrm{Se}$ & Shrub & Dec, Pow & oral & (Adjanohoun et al., 1991) \\
\hline & Hibiscus sabdariffa L. & Anyaba & 4431 & 0,09 & Lf & Shrub & Dec. & oral & (Tra Bi et al., 2008) \\
\hline & Sida linifolia Juss. & Odoe-ogbogbo & 4488 & 0,09 & Le & Herb & Dec. & Oral & \\
\hline \multirow[t]{3}{*}{ Meliaceae } & Azadiractha indica A.Juss. & Kiniti & 04647TgClt/AK & 0,09 & $\mathrm{Lf}$ & Tree & Dec. & Oral, & (Tra Bi et al., 2008) \\
\hline & Khaya senegalensis A. Juss. & Mahoghen & 4674 & 0,09 & Le ; St & Tree & Dec. & oral & (Karou et al., 2011) \\
\hline & $\begin{array}{l}\text { Pseudocedrela kotschy i(Schweinf.) } \\
\text { Harms }\end{array}$ & Yotsa & 7719 FDS/UL & 0,05 & $\mathrm{Bk}$ & Tree & Dec. & Oral & (Karou et al., 2011) \\
\hline \multirow[t]{2}{*}{ Moraceae } & Ficus exasperate Vahl & Sampepa & Togo 05094 & 0,05 & Rt & Herb & Dec & oral & (N'Guessan et al., 2009) \\
\hline & $\begin{array}{l}\text { Artocarpus altilis } \quad \text { (Parkinson) } \\
\text { Fosberg }\end{array}$ & Bere fruit & - & 0,09 & $\mathrm{Bk}$ & Tree & Dec. & Oral, & (Adjanohoun et al., 1991) \\
\hline Moringaceae & Moringa oleifera Lam. & Yovovitsi & 05250TG Clt/AK & 0,09 & $\mathrm{Le}$ & Tree & Dec. & oral & $\begin{array}{l}\text { (N'Guessan et al., } 2011 \text {; } \\
\text { Karou et al., 2011) }\end{array}$ \\
\hline Musaceae & Musa paradisiacal $\mathrm{L}$. & Banane & 1043FDS/UL & 0,05 & $\mathrm{Pw}$ & Tree & Dec. & oral & (Adjanohoun et al., 1991) \\
\hline Myrtaceae & Psidium guajava $\mathrm{L}$. & Gbèbèti & 470FDS/UL & 0,23 & Lf & Tree & Dec. & Oral & $\begin{array}{l}\text { (Karou et al., } 2011 \text {; Tra Bi et } \\
\text { al., 2008) }\end{array}$ \\
\hline & Syzygium guinensis (Willd.) DC. & Igiaro & & 0,05 & Rt & Herb & Dec. & Oral & (Adjanohoun et al., 1991) \\
\hline $\begin{array}{l}\text { Nephrolepidacea } \\
e\end{array}$ & $\begin{array}{l}\text { Nephrolepis undulate (Afzel. Ex Sw.) } \\
\text { J. Sm. }\end{array}$ & $\begin{array}{l}\text { Fougère de } \\
\text { palmier }\end{array}$ & Togo 12492 & 0,05 & Le & Tree & Dec. & oral & (Adjanohoun et al., 1991) \\
\hline Nyctaginaceae & $\begin{array}{l}\text { Boerhavia diffusa Engelm. \&A.Gray } \\
\text { L. }\end{array}$ & Ahozemeklo & Togo 05309 & 0,47 & $\mathrm{Rh}$ & Tree & Dec. & Oral, & (N’Guessan et al., 2009) \\
\hline \multirow{5}{*}{$\begin{array}{l}\text { Opiliaceae } \\
\text { Rubiaceae }\end{array}$} & Boerhaviaerecta $L$. & Babakou & 5319 & 0,09 & Lf & Shrub & Dec. & Oral, & (N’Guessan et al., 2009) \\
\hline & Opilia amentacea roxb. & Méfiodudami & 5525 & 0,09 & Lf & Herb & Dec. & oral & (Karou et al., 2011) \\
\hline & Sarcocephalus latifolius Sm. & Nyimon & 7536 & 0,09 & Rt & Tree & Dec. & oral & (Tra Bi et al., 2008) \\
\hline & Gardenia ternifolia Schumach. & Kawouti & 7361 & 0,09 & WP & Herb & Dec & oral & (Karou et al., \\
\hline & $\begin{array}{l}\text { Morinda lucida Benth. } \\
\text { Oxyanthus speciosus DC. } \\
\text { subsp. stenocarpus (K. Schum.) } \\
\text { Bridson }\end{array}$ & Zanklan & $\begin{array}{l}\text { Togo 07503/7497 } \\
-\end{array}$ & $\begin{array}{l}0,09 \\
0,14\end{array}$ & $\begin{array}{l}\text { Rt } \\
\text { Lf }\end{array}$ & $\begin{array}{l}\text { Tree } \\
\text { Herb }\end{array}$ & $\begin{array}{l}\text { Dec. } \\
\text { Cru }\end{array}$ & $\begin{array}{l}\text { oral } \\
\text { Cataplasm }\end{array}$ & $\begin{array}{l}\text { Tokoudagba et al., 2009) } \\
\text { (Karou et al., 2011) } \\
\text { (Adjanohoun et al., 1991) }\end{array}$ \\
\hline
\end{tabular}




\begin{tabular}{|c|c|c|c|c|c|c|c|c|c|}
\hline \multirow[t]{6}{*}{ Rutaceae } & $\begin{array}{lll}\text { Citrus } & \text { aurantifolia } & \text { (Christm.) } \\
\text { Swingle } & & \end{array}$ & Dontsi & Togo 02480 & 0,05 & Fr & Tree & Jce & Oral, & (N’Guessan et al., 2009) \\
\hline & Citrus aurantium $\mathrm{L}$. & N'ti & 782FDS/UL & 0,09 & Lf & Tree & Tea & Oral & (Karou et al., 2011) \\
\hline & Citrus grandis Hassk. & Azongbo & 44FDS/UL & 0,05 & $\mathrm{Fr}$ & Tree & Dec & Oral & (Karou et al., 2011) \\
\hline & $\begin{array}{l}\text { Clausena anisata (Willd.) Hook.f. ex } \\
\text { Benth. }\end{array}$ & Eyra & 08028TGClt/AK & 0,05 & Ro & Shrub & Dec & Oral & (Adjanohoun et al., 1991) \\
\hline & Fagara macrophylla Engl. & Ehéti & 8055 & 0,09 & $\mathrm{Lf}$ & Tree & Dec & oral & (N'Guessan et al., 2011) \\
\hline & $\begin{array}{l}\text { Zanthoxylum zanthoxyloides (Lam.) } \\
\text { Zepern. \& Timler }\end{array}$ & Ganhopovi & - & 0,14 & St & Tree & Pow & Massage & (Adjanohoun et al., 1991) \\
\hline Sapindaceae & Blighia sapida C. König & achanti & 8087 & 0,09 & $\mathrm{Lf}$ & Tree & Dec. & Oral, & (N'Guessan et al., 2011) \\
\hline Sapotaceae & Vitellaria paradoxa C.F.Gaertn. & Somou & 205FDS/UL & 0,19 & Ro & Tree & Tea & Bath & (Karou et al., 2011) \\
\hline \multirow[t]{3}{*}{ Solanaceae } & Schwenkia americana $\mathrm{L}$. & Kotoka & 8558 & 0,09 & $\mathrm{Fl}$ & Tree & Dec. & Oral & (Karou et al., 2011) \\
\hline & Solanum ethiopicum L. & Agbissan & 8519 & 0,09 & Fr & Herb & Dec. & Oral & (Karou et al., 2011) \\
\hline & $\begin{array}{l}\text { Solanumlycopersicum } \\
\text { (lycopersicumesculentus) }\end{array}$ & Timati & - & 0,09 & $\mathrm{Fr}$ & Shrub & $\mathrm{Cru}$ & Oral & (Karou et al., 2011) \\
\hline \multirow[t]{4}{*}{ Sterculiaceae } & Cola millenii K. Schum & Kpandotsi & Togo 08606 & 0,05 & Le & Tree & Dec & Oral & (Karou et al., 2011) \\
\hline & Cola nitida Schott \& Endl. & Goro & 8612 & 0,09 & $\mathrm{Le}$ & Tree & Dec & Oral & $\begin{array}{l}\text { (Karou et al., } 2011 \text {; Tra Bi et } \\
\text { al., 2008) }\end{array}$ \\
\hline & Theobroma cacao L. & Coco & 8669 & 0,09 & $\mathrm{Se}$ & Tree & Dec. & Oral & (Karou et al., 2011) \\
\hline & Waltheria indica $\mathrm{L}$. & - & 8678 & 0,09 & Rt & Tree & Dec. & Oral & $\begin{array}{l}\text { (Adjanohoun et al., } 1991 \text {; } \\
\text { Cisse et al., 2016) }\end{array}$ \\
\hline Ulmaceae & $\begin{array}{l}\text { Trema guineensis (Schumach. } \\
\text { \&Thonn.) Ficalho }\end{array}$ & Waza - Waza & 08974TGClt/AK & 0,05 & WP & Shrub & Dec. & Oral & (Adjanohoun et al., 1991) \\
\hline \multirow[t]{3}{*}{ Verbenaceae } & Gmelina arborea Roxb. & Gboviti & - & 0,09 & Lf & Herb & Dec & oral & (Karou et al., 2011) \\
\hline & Lippia multiflora Moldenke & Avondati & 09207TG Clt/AK & 0,05 & Lf & Shrub & Tea & oral & (Adjanohoun et al., 1991) \\
\hline & $\begin{array}{l}\text { Stachytarpheta angustifolia Mill. } \\
\text { Vahl }\end{array}$ & $\begin{array}{l}\text { Tchoumboulouz } \\
\text { ou }\end{array}$ & 9255 & 0,09 & Rt & Herb & Dec. & Oral & (Karou et al., 2011) \\
\hline \multirow[t]{2}{*}{ Zingiberaceae } & Aframomum melegueta K.Schum. & Atakun & 204FDS/UL & 0,05 & $\mathrm{Se}$ & Herb & Dec, Tea & Oral, & (Adjanohoun et al., 1991) \\
\hline & Zingiber officinale Roscoe & Dotè & 348FDS/UL & 0,05 & $\mathrm{Rh}$ & Shrub & Tea & Oral & (Adjanohoun et al., 1991) \\
\hline
\end{tabular}

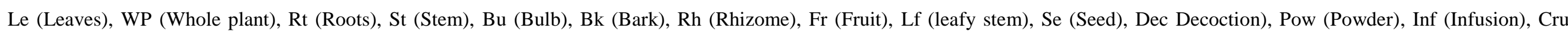
(Crushing), Pow (powder). 


\section{Discussion}

The present survey was undertaken to identify medicinal plants used in the south of Togo for the treatment of hypertension. We were first interested in the mode diagnosis of the disease by the TH. The results revealed that all the surveyed TH were able to cite the mains symptoms. We observed that severe headache, severe anxiety, shortness of breath, nosebleed, Loss of consciousness and fear of heights were commonly used by TP to diagnose the disease before the treatment. However the risk of misdiagnosis remains, as very few of them collaborate with the modern medicine. In fact, some authors reported the problems of misdiagnosis by traditional healers for some diseases that were not common in African areas. These diseases often called emerging diseases in Africa included cancers and metabolic diseases like hypertension (Alonso-Castro et al., 2011; Taylor et al., 2014).

Different plants species were used by Togolese TH to heal hypertension attack. There were 116 species of plants with the Fabaceae family as the most dominant with 16 species. In the same context, a previous study was carried out in the central region of the Togo (Karou et al., 2011). The study enabled the identification of 38 antihypertensive recipes; while in a similar study Tsabang et al. (2016) identified 71 recipes both treating arterial hypertension and diabetes in Cameroon. The recipes from Togolese TH were made from 64 plant species in the flora of Togo. The most commonly cited as antihypertensive were Parkia biglobosa, Khaya senegalensis, Gardenia ternifolia, and Persea americana. In comparison with previous published reports, the THs of the Togo Central Region were found to have basic knowledge regarding herbal medicine for the treatment of hypertension [19]. Investigations on medicinal plants in the markets of Abidjan allowed the inventory of 58 species of plants used to treat 19 current diseases (N'Guessan et al., 2009). Thirty-nine of these plants were used against arterial hypertension and diabetes. These plants were Catharanthus roseus (L.) G Don, Ageratum conyzoides L, Vernonia colorata (Willd.) Drake, Alchornea cordifolia (Schum.\& Thonn.)Müll.Arg., Phyllanthus amarus Schum.\& Thonn.and Parkia biglobosa (Jacq.) Benth (N'Guessan et al., 2009). In morocco an ethnobotanical surveys allowed an inventory of 57 medicinal plant species belonging to 52 genera and 30 families. Of these, 40 are traditionally used against diabetes and 30 against hypertension (Ziyyat et al., 1997; Tahraoui et al., 2007).

In Benin a neighboring country of Togo, ethnobotanical surveys were carried out with 36 trait-therapists from the Bassila district using individual interviews. A total of 23 plant species belonging to 16 botanical families were reported to have antihypertensive properties. The most cited species were Parkia biglosa, Allium sativum, Allium cepa and Cassia sieberiana. The root was the most widely used plant organ and the decoction was the main mode of preparation of medicinal products which are generally administered orally (Anselme Bio et al., 2005). Another ethnopharmacological studies have identified medicinal plants used for their antihypertensive properties of which eleven were harvested and tested for the vasodilatory activity. Two species namely Parkia biglobosa and Spondias mombin displayed promising results (Anselme Bio et al., 2005).

In the present study the most important plants were Byrsocarpus coccineus, Crateva religiosa and Boerhavia diffusa on the basis of the use value. These three plants were already cited in the literature for similar use in the traditional medicine. Moreover, Dada et al. screened Byrsocarpus coccineus for its antihypertensive activity and found an interesting activity of the hydroethanolic leaf extract in relation with the antioxidant property and improvement of lipid profile (Dada et al., 2013).

\section{Conclusion}

This survey provides initial evidence of the use of plants by Togolese traditional medicine practitioners to manage Hypertension and this by family inheritance in traditional initiation manner, as the species were cited at least once in previous ethnobotanical reports. It is therefore necessary for scientist to go further in characterization of the biomolecules. The present inventory therefore represents the contribution of natural flora of Togo to the global approach in controlling hypertension.

Acknowledgement: The authors gratefully thank all the traditional healers of the maritime region who accepted to participate to the study.

Conflict of interest: The authors declare no conflict of interest.

\section{References}

1. Aburjai T, Hudaib M, Tayyem R, Yousef M, Quishawi M (2007). Ethnopharmacological survey of medicinal herbs in Jordan, the Ajloun Eights region. Journal of Ethnopharmacology. 76: 99-103.

2. Adjanohoun E, Ahiyi MRA, Ake Assi L, Dramane M, Elewude JA, Fadoju SU, Gbile ZO, Goudote E, Johnson CLA, Keita A, Morakinyo O, Ojewole JAO, Olatunji AO, Sofowora EA. (1991). Contribution to Ethnobotanical and floristic studies in westen Nigeria, CSTR/OUA. 420 p.

3. Adodo A (2004).Nature Power: A Christian Approach to Herbal Medicine: Generation Press; p. 304.

4. Ajibesin KK, Ekpo BA, Bala DN, Essien EE, Adesanya SA (2008). Ethnobotanical survey of Akwa Ibom State of Nigeria. Journal of Ethnopharmacology. 115:387-408. 
5. Alonso-Castro AJ, Villarreal ML, Salazar-Olivo LA, Gomez-Sanchez M, Dominguez F, Garcia-Carranca A (2011). Mexican medicinal plants used for cancer treatment: Pharmacological, phytochemical and ethnobotanical studies. Journal of Ethnopharmacology. 133: 945-972.

6. Anselme B, Mireille TS, Yoka J, Djego GJ, Awede B, Laleye A, Sinsin AB (2015). Contribution aux connaissances des principales plantes antihypertensives utilisées en médecine traditionnelle à Bassila (Bénin, Afrique de l'Ouest). Revue du CAMES. Série Pharmacopée et médecine traditionnelle africaine. 17.

7. Atanasov AG, Waltenberger B, Pferschy-Wenzig EM, Linder T, Wawrosch C, Uhrin P, Temml V, Wang L, Schwaiger S, Heiss EH, Rollinger JM, Schuster D, Breuss JM, Bochkov V, Mihovilovic MD, Kopp B, Bauer R, Dirsch VM, Stuppner H. (2015). Discovery and resupply of pharmacologically active plant-derived natural products: A review. Biotechnology. Advances. 08: 001.

8. Baharvand-Ahmadi B, Asadi-Samani M (2016). A mini-review on the most important effective medicinal plants to treat hypertension in ethnobotanical evidence of Iran. Journal of Nephropharmacology. 6:3-8.

9. Baharvand-Ahmadi B, Bahmani M, Tajeddini P, Rafieian-Kopaei M, Naghdi N (2016). An ethnobotanical study of medicinal plants administered for the treatment of hypertension. Journal of Renal Injury Prevention. 5:123-8.

10. Briskin DP (2000). Medicinal Plants and Phytomedicines. Linking Plant Biochemistry and Physiology to Human Health. Plant Physiology. 124: 507-514.

11. Cisse A, Gueye M, KA A, Ndiaye F, Koma S, Akpo LE (2016). Ethnobotanique des plantes médicinales chez les bergers peuls de WidouThiengoly de la commune de Téssékéré (Ferlo-Nord Sénégal). Journal of Applied Biosciences. 98:9301-9308.

12. Cohen KA, Abeel T, Manson-McGuire A, Desjardins CA, Munsamy V, Shea TP, Walker BJ, Bantubani N, Almeida DV, Alvarado L, Chapman SB, Mvelase NR, Duffy EY, Fitzgerald MG, Govender P, Gujja S, Hamilton S, Howarth C, Larimer JD, Maharaj K, Pearson MD, Priest ME, Zeng Q, Padayatchi N, Grosset J, Young SK, Wortman J, Mlisana KP, O'Donnell MR, Birren BW, Bishai WR, Pym AS, Earl AM. (2015). Evolution of Extensively Drug-Resistant Tuberculosis over Four Decades: Whole Genome Sequencing and Dating Analysis of Mycobacterium tuberculosis Isolates from KwaZulu-Natal. PLoS Medicine. 12:e1001880.

13. Dada OK, Akindele AJ, Morakinyo OA, Sofidiya MO, Ota D (2013). Hypoglycemic and antioxidant activities of the hydroethanolic leaf extract of Byrsocarpus coccineus Schumach. \& Thonn. (Connaraceae). Chinese Journal of Natural Medicines. 11:628-37.

14. Dibong SD, Mpondo Mpondo E, Ngoye A, Kwin NF, Betti JL (2011). Ethnobotanique et phytomédecine des plantes médicinales vendues sur les marchés de Douala, Cameroun. Journal of Applied Biosciences. 37: 2496-2507.

15. Dzib-Guerra WD, Escalante-Erosa F, García-Sosa K, Derbré S, Blanchard P, Richomme P, Peña-Rodríguez LM (2016). Anti-Advanced Glycation End-product and Free Radical Scavenging Activity of Plants from the Yucatecan Flora. Pharmacognosy Research. 8:276-280.

16. Frohlich ED (2001). Preventive cardiology: more than just lipid lowering. Preventive Cardiology Journal. 4:101-102.

17. Gali-Muhtasib H, Hmadi R, Kareh M, Tohme R, Darwiche N (2015). Cell death mechanisms of plant-derived anticancer drugs: beyond apoptosis. Apoptosis. 20:1531-62.

18. Hudaib M, Mohammad M, Bustanji Y, Tayyem R, Yousef M, Abuirjeie M, Aburjai T. (2008). Ethnopharmacological survey of medicinalplants in Jordan, Mujib Nature Reserve and surrounding area. Journal of Ethnopharmacology. 120: 63-71.

19. Ilboudo DP, Basilico N, Parapini S, Corbett Y, D’Alessandro S, Dell'Agli M, Coghi P, Karou SD, Sawadogo R, Gnoula C, Simpore J, Nikiema JB, Monti D, Bosisio E, Taramelli D. (2013). Antiplasmodial and anti-inflammatory activities of Canthium henriquesianum (K. Schum), a plant used in traditional medicine in Burkina Faso. Journal of Ethnopharmacology. 148: 763-769.

20. Karou SD, Tchacondo T, Tchibozo MAD, Abdoul-Rahaman S, Anani K, Koudouvo K, Batawila K, Agbonon A, Simpore J, de Souza C. (2011). Ethnobotanical study of medicinal plants used in the management of diabetes mellitus and hypertension in the Central Region of Togo. Pharm Biol. 49: 1286-1297.

21. Kayode J (2006). Conservation of indigenous medicinal botanicals in Ekiti State, Nigeria. Journal of Zhejiang University Science B. 7:713-8.

22. Larremore DB, Sundararaman SA, Liu W, Proto WR, Clauset A, Loy DE, Speede S, Plenderleith LJ, Sharp PM, Hahn BH, Rayner JC, Buckee CO. (2015). Ape parasite origins of human malaria virulence genes. Nature Communications. 6: 8368 .

23. Le Doare K, Bielicki J, Heath PT, Sharland M (2015). Systematic Review of Antibiotic Resistance Rates among GramNegative Bacteria in Children with Sepsis in Resource-Limited Countries. Journal of the Pediatric Infectious Diseases Society. 4: 11-20.

24. N'Guessan H A, Dago D C, Mamyrbékova-Békro J A, Békro YA (2011). Chromatographie sur Couche Mince d'extraits sélectifs de 10 plantes utilisées dans le traitement traditionnel de l'hypertension artérielle en côte d'ivoire. European Journal of Scientific Research.66 (4), pp. 575-585

25. N'Guessan K, Kadja B, Zirihi NG, Traoré D, Aké-Assi L (2009). Screening phytochimique de quelques plantes médicinales ivoiriennes utilisées en pays Krobou (Agboville, Côte-d'Ivoire). The Science of Nature. Vol. $6 \mathrm{~N}^{\circ} 1$ : $1-$ 15.

26. Oneko M, Kariuki S, Muturi-Kioi V, Otieno K, Otieno VO, Williamson JM, Folster J, Parsons MB, Slutsker L, Mahon BE, Hamel MJ. (2015). Emergence of Community-Acquired, Multidrug-Resistant Invasive Nontyphoidal Salmonella Disease in Rural Western Kenya, 2009-2013. Clinical Infectious Diseases. 61 Suppl 4: S310-316. 
27. Orch H, Douira A, Zidane L (2015). Étude ethnobotanique des plantes médicinales utilisées dans le traitement du diabète, et des maladies cardiaques dans la région d'Izarène (Nord du Maroc). Journal of Applied Biosciences. 86:7940- 7956.

28. Padal SB, Prayaga Murty P, Srinivasa DR, Venkaiah M (2010). Ethnomedicinal plants from Paderu division of Visakhapatnam district, A.P, India. Journal of Phytology. 2: 70-91.

29. Rafieian-Kopaei M. (2011). Medicinal plants and the human needs. Journal of Biology. 35: 635-9.

30. Rosa TFA, Flammersfeld A, Ngwa CJ, Kiesow M, Fischer R, Zipfel PF, Skerka C, Pradel G. (2016). The Plasmodium falciparum blood stages acquire factor $\mathrm{H}$ family proteins to evade destruction by human complement.Cellular Microbiology. 18 (4):573-90.

31. Rouhi-Boroujeni H, Heidarian E, Rouhi-Boroujeni H, Deris F, Rafieian-Kopaei M (2017). Medicinal plants with multiple effects on cardiovascular diseases: A systematic review. Current Pharmaceutical Design. 23(7):999-1015.

32. Sereme A, Millogo-Rasolodimby J, Guinko S, Nacro M (2008). Proprietes Therapeutiques Des Plantes A Tanins Du Burkina Faso. Revue du CAMESPharmacopée et Médecine Traditionnelle Africaines. 15: 41 - 49.

33. Sowers JR, Whaley-Connell A, Epstein M (2009). Narrative review: the emerging clinical implications of the role of aldosterone in the metabolic syndrome and resistant hypertension. Annals of Internal Medicine. 150:776-83.

34. Svilaas A, Strandberg T, Eriksson M, Hildebrandt P, Westheim A (2008). Lipid lowering treatment patterns and goal attainment in Nordic patients with hyperlipidemia. Scandinavian Cardiovascular Journal 42:279-87.

35. Tahraoui A, El-Hilaly J, Isrili ZH, Lyoussi B (2007). Ethnopharmacological survey of plants used in the traditional treatment of hypertension and diabetes in South Eastern Morocco (Errachidia Province). Journal of Ethnopharmacology. 110: 105-117.

36. Taylor P, Colman L, Bajoon J (2014). The search for plants with anticancer activity: Pitfalls at the early stages. Journal of Ethnopharmacology. 158: 246-254.

37. Tchacondo T, Karou DS, Batawila K, Agban A, Ouro-Bang'na K, Anani KT, Gbeassor M, de Souza C (2011). Herbal remedies and their adverse effects in tem tribe traditional medicine in Togo. African Journal of Traditional, Complementary and Alternative medicines. 8: 45-60.

38. Tchacondo T, Karou SD, Agban A, Bako M, Batawila K, Bawa ML, Gbeassor M, de Souza C. (2012). Medicinal plants use in central Togo (Africa) with an emphasis on the timing. Pharmacognosy Research. 4: $92-103$.

39. Tokoudagba JM, Chabert P, Auger C, NGom S, Gbenou J, Moudachirou M, Schini-Kerth V, Lobstein A. (2009). Recherchedeplantesàpotentialités antihypertensivesdanslabiodiversitébéninoise. Ethnopharmacologia 44 : 32-41.

40. Tra Bi FH, Irie GM, N'Gaman K, Mahou CHB (2008). Études de quelques plantes thérapeutiques utilisées dans le traitement de l'hypertension artérielle et du diabète : deux maladies émergentes en Côte d'Ivoire. The Science of Nature. 5 : pp. 39-48.

41. Tsabang N, Tsambang DWL, Tsambang FSC, Agbor AG (2016). Ethnomedical and ethnopharmacological study of plants used for potential treatments of diabetes and arterial hypertension by indigenous people in three phytogeographic regions of Cameroon. Diabetes Case Reports. 2016,1:2.

42. Ziyyat A, Legssyer A, Mekhfi H, Dassouli A, Serhrouchni M, Benjelloun W (1997). Phytotherapy of hypertension and diabetes in oriental Morocco. Journal of Ethnopharmacology. 58: 45-54. 